\title{
A RARE CASE OF ANTERIOR NASAL SEPTAL CHONDROMA
}

\author{
Kurian Ninan, Rahul Shirol, Chethan T. K.
}

1. Assistant Professor. Department of Radiodiagnosis, K. S. Hegde Medical Academy, Deralakatte, Mangalore.

2. Post Graduate. Department of Radiodiagnosis, K. S. Hegde Medical Academy, Deralakatte, Mangalore.

3. Post Graduate. Department of Community Medicine, K. S. Hegde Medical Academy, Deralakatte, Mangalore.

\section{CORRESPONDING AUTHOR:}

Dr. Rahul Shirol,

3-474(3) Kamath compound,

Kuthar Padavu, Munnur,

Mangalore- 575017.

E-mail: rahul_shirol@yahoo.com

Ph: 00919844284171

ABSTRACT: Chondromas of the nasal septum are rare. Since its first literary description in 1842 , only about 141 cases have been reported. I like to stress on a rare type of lesion, ie; chondroma, which can affect nasal septum. I will be discussing the pattern of involvement of the nasal septum by the lesion through a case involving a 45 year old woman who presented with one year of nasal obstruction which was more on the left side. A pale mass arising from the nasal septum was found in the left nasal cavity. The tumor was removed under Functional endoscopic sinus surgery and histopathologic examination revealed showed hyaline cartilage cells. Despite their rarity, chondromas should be taken into consideration in the differential diagnosis of nasal tumors, especially those arising from the nasal septum.

KEY WORDS: Nasal septum, chondromas

INTRODUCTION: Cartilaginous tumors of the head and neck are rare. The most frequent sites that the cartilaginous tumors are usually observed are the larynx and the spheno-ethmoidal area. Chondroma is a benign cartilaginous neoplasm and is rarely seen in the nasal septum. Including this case, the number of reported nasal septal chondromas is only 141 cases even though the first description in the literature was done in 1842. ${ }^{1}$. Chondrogenic tumors are relatively uncommon, with malignancy occurring twice as frequently as in benign tumors ${ }^{2}$. Only few cases have been reported to be arising from the anterior part of the nasal septum. Considering the rarity, this report will document a solitary chondroma of the nasal septum with its origin from the anterior cartilaginous part. There will be a discussion about the problems encountered in the radiological interpretation of this tumor.

CASE REPORT: A 45 year old woman presented with one year insidious onset of nasal obstruction which was predominately more on the left side. There was change in voice since a year and also had six months of intermittent headache. Clinical examination revealed a fleshy mass filling the left nasal cavity in the middle meatus. The mass was pale, non tender and did not bleed. The cervical lymph nodes were not palpable.

Unenhanced CT scan of the nasal cavity and the paranasal sinuses showed a midline soft tissue density lesion in the anterior aspect of the nasal cavity causing bowing and scalloping of the medial walls of both maxillary sinuses as well as the hard palate (Fig 1). No intra-tumoral calcification or cavitations seen. Post contrast study showed minimal enhancement. Posterior 
nasal septum appeared intact. However there is destruction of the floor of the sphenoid sinus with extension of the lesion into the same (Fig 2). Cervical lymphadenopathy was not visualized.

Functional endoscopic sinus surgery was performed. The red jelly like mass was sent for histopathological interpretation. It showed hyaline cartilage cells arranged in lobules. No hyperchromasia or nuclear atypia was found. There was no evidence of local recurrence during the six month followup.

DISCUSSION: Overall, the incidence of chondroma peaks during the sixth decade of life 3 . However, in the facial skeleton, chondroma generally occurs during adolescence and early adulthood ${ }^{4}$ unlike this case. The most common reported sites in the facial skeleton are the ethmoid sinuses and the nasal cavity excluding the nasal septum (incidence: 50\%); other common sites are the nasal septum $(17 \%)$, the hard palate $(6 \%)$, and the nasopharynx $(6 \%)^{5}$. They are usually well circumscribed and appear fairly homogenous on CT. They tend to be expansile lesions that remodel bone ${ }^{6}$. They do not provoke sclerotic bone at their margins. Calcification of the chondroid matrix occurs rarely ${ }^{7}$. All these features were consistent with this case. However destruction of the floor of the sphenoid sinus with extension into the same showed an aggressive side to it.

Chondrogenic tumors of the facial skeleton also show aggressive behavior. Because of the discrepancy between the histological picture and biologic behavior, chondrogenic neoplasm should be considered potentially malignant 7 . Hence wide surgical excision should be considered as treatment of choice.

The differential diagnosis includes Wegener's granulomatosis nasal polyps, fungal, rhinosporidiosis, infections and tuberculosis. Nasal polyps are the most common expansile lesions in the nasal cavity. But the negative history of vasomotor rhinitis and allergy along with radiological features like bowing of the surrounding bones with destruction of the nasal septum does not go in favour. Similarly the other granulomatous and fungal lesions have variable degrees of bone destruction. However, the clear educational message here is that when there is a midline lesion showing scalloping or destruction of surrounding bones, nasal chondroma must also be considered as a differential diagnosis.

\section{REFERENCES:}

1. Scierski W, Namysłowski G, Misiołek M, Widziszowska A, Hajduk A. Septal nasal chondroma--a case report. Otolaryngol Pol. 2007;61(6):1021-3

2. Murthy DP, Gupta AC, SenGupta SK, Dutta TK, Pulotu ML. Nasal cartilaginous tumour. J Laryngol Otol 1991;105: 670 -672

3. Batsakis JG. Tumors of the Head and Neck: Clinical and Pathological Considerations. 2nd ed. Baltimore: Williams \& Wilkins, 1979:219-20, 383-7

4. Faccini JM, Williams JL. Nasal chondroma. J Larnyngol Otol 1973;87:811-16.

5. Kilby D, Ambegaokar AG. The nasal chondroma. 2 case reports and a survey of the literature. J Larnyngol Otol 1977; 91:415-26.

6. Som PM. Sinonasal Cavity. In: Som PM, Bergeron RT, eds. Head and neck imaging, 2nd ed. St. Louis: Mosby, 1991: 114-200

7. Ruark DS, Schlehaider VK, Shah JP. Chondrosarcomas of the head and neck. World J Surg 1992;16: 1010 -1016 
CASE REPORT

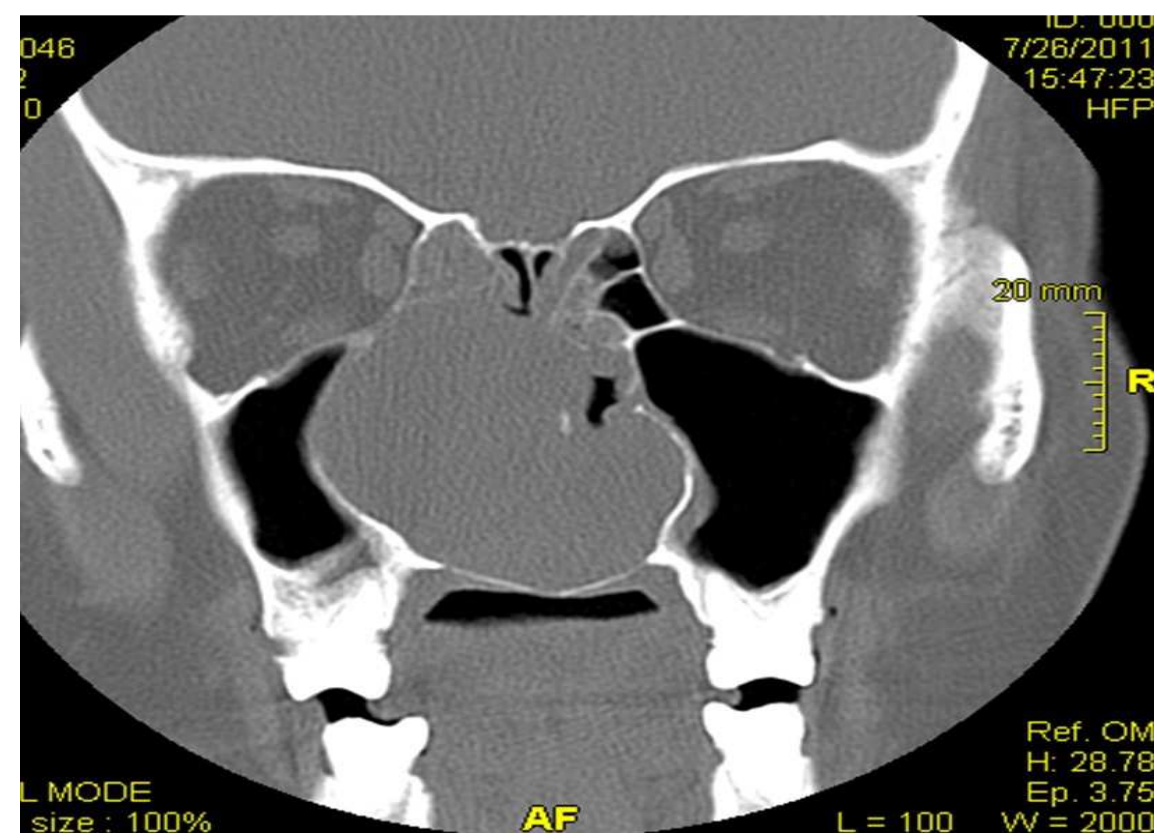

FIGURE 1: Bowing and scalloping of the medial walls of both maxillary sinuses

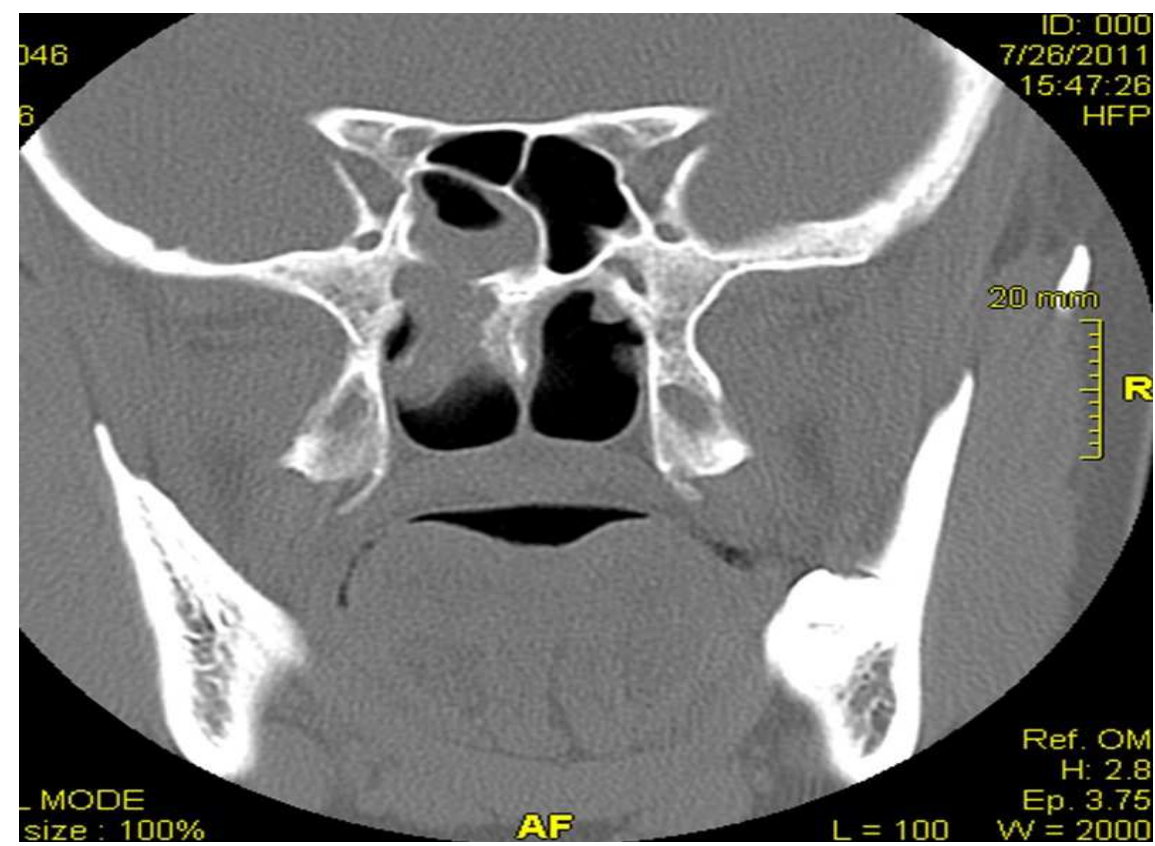

FIGURE 2: Destruction of the floor of the sphenoid sinus. 